\title{
Analysis of ileostomy stool samples reveals dysbiosis in patients with high-output intestinal fluid
}

\author{
Hirokazu Matsuzawa \\ Juntendo University \\ Shinya Munakata ( $\nabla$ smunaka@juntendo.ac.jp ) \\ Juntendo University https://orcid.org/0000-0002-2771-2804 \\ Masaya Kawai \\ Juntendo University \\ Kiichi Sugimoto \\ Juntendo University \\ Hirohiko Kamiyama \\ Juntendo University \\ Makoto Takahashi \\ Juntendo University \\ Yutaka Kojima \\ Juntendo University \\ Kazuhiro Sakamoto \\ Juntendo University
}

\section{Research article}

Keywords: dysbiosis, high output, ileostomy, rectal cancer

Posted Date: April 2nd, 2020

DOI: https://doi.org/10.21203/rs.3.rs-17781/v1

License: (c) (1) This work is licensed under a Creative Commons Attribution 4.0 International License.

Read Full License 


\section{Abstract}

Background Diverting stoma construction can significantly reduce the onset of severe anastomotic leakage in patients with rectal cancer. High-output stoma is one of the most important potential surgical complications after anal function-preserving surgery with ileostomy. Culture-independent techniques have revealed the interaction of complex intestinal bacterial ecology with various diseases. Our objective was to evaluate the differences in patient characteristics and gut microbiota distribution features in stoma high output patients.

Patients and Methods The cases of 24 consecutive patients who underwent curative resection for rectal cancer at our hospital between November 2016 and June 2018 were reviewed and patients categorized into high-output and low-output groups. The microbiota was analyzed using next-generation sequencing of ileostomy stool samples collected on postoperative day 7. Results There was a significant difference in the percentage of Bacteroidetes between the high-output and low-output groups ( $14.8 \%$ vs $0.5 \% ; p=$ $0.01)$. The percentage of Clostridium butyricum was increased in the low-output group $(p=0.01)$. The analyses after the exclusion of those treated with the Miya-BM probiotic, whose principal component is $\mathrm{C}$. butyricum, revealed no significant differences between the high-output and low-output groups.

Conclusion This pilot study provides first evidence correlating gut microbiota in the pathogenesis of high output stoma compared with low output stoma.

\section{Background}

Diverting stoma construction is useful in significantly reducing the onset of severe anastomotic leakage $(A L)$ that requires additional surgery in patients undergoing low anterior resection (LAR) or intersphincteric resection (ISR) for rectal cancer. ${ }^{1}$ Although one of the most important potential surgical complications after anal function-preserving surgery is $\mathrm{AL}$, which can result in morbidity and/or mortality, stoma-related complications such as stoma infection, obstructive complications, and electrolyte imbalance can also lead to severe problems. ${ }^{2}$ High-output ileostomy may result from partial or intermittent bowel obstruction.

High stoma output is characterized by an increased loss of fluids and sodium through fecal drainage, which may lead to hyponatremia, dehydration, and hyperaldosteronism. ${ }^{3}$ Studies have reported that the majority of patients with high stoma output presented with only moderate decreases in glomerular filtration rate that were not clinically significant; however, $30 \%$ of the patients were found to have renal failure secondary to dehydration, requiring readmission. ${ }^{3,4}$ Patients with a daily stomal loss of $<1200 \mathrm{~mL}$ can usually maintain sodium balance by adding extra salt to the limit of palatability at the table and when cooking. When the stomal loss is in the range of $1200-2000 \mathrm{~mL}$, or sometimes more, patients are more likely to have issues related to dehydration due to water and sodium loss through sweat, especially in hot weather. ${ }^{5}$ 
Culture-independent techniques for bacterial identification based on 16S ribosomal RNA (rRNA) sequences have revolutionized the understanding of the complex intestinal bacterial ecology associated with various diseases. ${ }^{6,7}$

Despite advances in lower rectal surgery to preserve the sphincter and prevent AL using ileostomy, the consequences of stoma-related dehydration may be underestimated. The pathogenesis underlying highoutput stoma is unclear. Therefore, the objective of the current study was to evaluate the differences in patient characteristics and the gut microbiota distribution in patients with high-output stomas.

\section{Methods}

The data of 24 consecutive patients who underwent curative resection for rectal cancer with ileostomy by a laparoscopic approach at Juntendo University Hospital between November 2016 and June 2018 were reviewed in this retrospective study. The inclusion criteria were stage $\nabla-\Downarrow$ cancer, lateral pelvic lymph node dissection, and previous treatment with neoadjuvant pelvic chemoradiotherapy and chemotherapy. Patients who underwent emergency surgery, those with synchronous cancers, and those who underwent abdominoperineal resection were excluded from the current study.

Most of the total and tumor-specific mesorectal excisions were performed by the same team of staff colorectal surgeons. By using ileum of $30-40 \mathrm{~cm}$ from ileum end, lleostomy were made on lower right abdominal wall. ileostomy stool samples were collected on postoperative day 7. Rectal anastomoses were performed using the double stapling technique in patients undergoing LAR. Reconstruction comprised hand-sewn coloanal anastomosis in patients undergoing ISR. In all patients, cancer staging was based on the eighth edition of the TNM classification of malignant tumors. The indications for loop ileostomy were the following: anastomosis $<5 \mathrm{~cm}$ from the anal verge, obstruction, neoadjuvant therapy, intraoperative technical issues, and severe diabetes mellitus. Preoperative neoadjuvant chemotherapy (such as FOLFOX or CapeOX) or chemoradiotherapy (such as S-1 with 45-Gy radiation) were

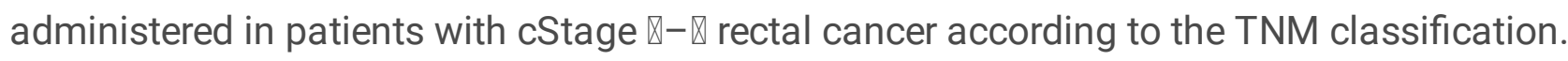

No chemical preparations such as those including kanamycin and metronidazole were administered preoperatively. ${ }^{8,9}$ All patients were intravenously administered $3 \mathrm{~g} /$ day cefmetazole, a cephamycin antibiotic. The use of probiotics, such as Miya-BM containing Clostridium butyricum M588, was based on the surgeon's discretion.

AL was defined by the following clinical criteria: pelvic abscess, fecal discharge from the wound and drain, septicemia, and peritonitis, with or without radiologically confirmed leakage. ${ }^{10}$ Grading of AL was performed as described by Rahbari et al. ${ }^{11}$ Surgical site infections were defined using the U.S. Centers for Disease Control definitions. ${ }^{12}$ Postoperative ileus was defined as the inability to tolerate food in the presence of abdominal distension, absence of bowel sounds, and the need to delay enteral feeding. ${ }^{13}$ Grading was based on the Clavien-Dindo classification. ${ }^{14}$ High output was defined as an ostomy output $\geq 1500 \mathrm{~mL}$, as described in previous studies. ${ }^{15,16}$ 


\section{DNA extraction from stool samples and 16S rRNA sequencing}

The ileal stool from the stoma pouch were suspended in $900 \mu \mathrm{L}$ buffer containing $4 \mathrm{M}$ guanidium thiocyanate, $100 \mathrm{mM}$ Tris- $\mathrm{HCl}(\mathrm{pH} 9.0)$, and $40 \mathrm{mM}$ ethylenediaminetetraacetic acid (pH 8.0) and centrifuged at $20800 \mathrm{~g}$ for $5 \mathrm{~min}$. The supernatants were discarded, and $600 \mu \mathrm{l} \mathrm{TE}$ buffer $(10 \mathrm{mmoL} / \mathrm{L}$ Tris- $\mathrm{HCl} \mathrm{pH} \mathrm{8.0,1} \mathrm{mmoL/L} \mathrm{ethylenediaminetetraacetic} \mathrm{acid} \mathrm{pH} \mathrm{8.0)} \mathrm{was} \mathrm{used} \mathrm{to} \mathrm{wash} \mathrm{the} \mathrm{pellets} \mathrm{twice.}$ Next, glass beads (diameter, $0.15-0.21 \mathrm{~mm}$ ) were added, and the samples were homogenized at 7000 rpm for 20 s using a MagNA Lyser instrument (Roche, Penzberg, Germany). Next, $2 \mu \mathrm{L}$ lysozyme (10 $\mathrm{mg} / \mathrm{mL}$; Wako, Osaka, Japan) was added, the samples were incubated for $1 \mathrm{~h}$ at $37^{\circ} \mathrm{C}$. Next, $100 \mu \mathrm{L}$ of $10 \%$ sodium dodecyl sulfate (Kanto Kagaku Tokyo, Japan) and $600 \mu \mathrm{L}$ of the phenol/chloroform/isoamyl alcohol solution (Nippon Gene, Tokyo, Japan) were added, and the samples were processed at $7000 \mathrm{rpm}$ for $20 \mathrm{~s}$ in the MagNA Lyser. Next, the samples were centrifuged at $20800 \mathrm{~g}$ for $5 \mathrm{~min}$, and $600 \mu \mathrm{l}$ of the supernatants were transferred to $1.5-\mathrm{mL}$ tubes, followed by the addition of $600 \mu \mathrm{L}$ isopropanol and $60 \mu \mathrm{L}$ of $3 \mathrm{M}$ sodium acetate (Nippon Gene). The samples were centrifuged at $20800 \mathrm{~g}$ for $5 \mathrm{~min}$, and the supernatants were removed by decanting. The DNA pellets were washed with $70 \%$ ethanol, dried with a centrifugal evaporator (Eyela, Tokyo, Japan), and dissolved by incubating in $200 \mu \mathrm{L}$ TE buffer and $2 \mu \mathrm{L}$ RNase ( $1 \mathrm{mg} / \mathrm{mL}$; Nippon Gene) for $1 \mathrm{~h}$. The purity of the samples were determined with the High PCR template preparation kit (Roche, Basel, Switzerland). The DNA concentrations were quantified using the QuantiFluor One dsDNA system (Promega, Wisconsin, USA).

The variable $\mathrm{V} 3-4$ regions of the $16 \mathrm{~S}$ rRNA gene were amplified by polymerase chain reaction using $341 \mathrm{~F}$ (50-AATGATACGGCGACCACCGAGATCTACAC(adaptor sequence) + barcode (eight bases)+ACACTCTTTCCCTACACGACGCTCTTCCGATCT (sequence primer) + CCTACGGGNGGCWGCAG30) and 805R primers (50-CAAGCAGAAGACGGCATACGAGAT(adaptor sequence)+ barcode (eightbases) + GTGACTGGAGTTCAGACGTGTGCTCTTCCGATCT (sequenceprimer) + GACTACHVGGGTATCTAATCC30)and the Takara Ex Taq ${ }^{\circledR}$ Hot Start Version kit (Takara, Otsu, Japan). The amplicons generated from the samples were purified using SPRIselect (Beckman Coulter, Pennsylvania, USA). The DNA concentrations of purified amplicons were quantified using the QuantiFluor One dsDNA system, and equal DNA amounts were pooled.

The pooled samples were sequenced using Miseq Reagent kit V3 (600-cycle; Illumina, San Diego, CA, USA) on the Miseq system according to the manufacturer's instructions. Sequence data were analyzed using the Quantitative Insights into Microbial Ecology (QIIME) pipeline (version1.9.1).

\section{Determination of a-diversity and $\beta$-diversity}

The number of observed species and Chao1 and Shannon phylogenetic diversity indices were calculated using the R package "phyloseq" and statistically analyzed using Wilcoxon's test. $\beta$ - diversity was 
estimated using the UniFrac metric to calculate the distances between the samples and visualized by principal coordinate analysis. The graphs were generated using QIIME pipeline (version 1.9.1).

\section{Statistical analysis}

The statistical software JMP version 13 (SAS Institute, Cary, N.C., USA) was used for all statistical analyses. Categorical variables were compared using the chi-squared or Fisher's exact test, as appropriate. Continuous variables were presented as medians and compared using the Mann-Whitney $U$ test or analysis of variance. Sequence data were presented as median \pm standard error of the mean (SEM). $P$ values $<0.05$ were considered to indicate statistical significance.

\section{Results}

Table 1 summarizes the clinicopathological characteristics of the 24 patients who underwent surgical resection for rectal cancer with ileostomy. The study cohort comprised 17 males and 7 females, with a median age of 63 (range, 37-82) years. Additionally, 8 (33.3\%) patients were treated with neoadjuvant chemotherapy and $1(4.1 \%)$ patient was treated with neoadjuvant chemoradiotherapy. LAR and ISR were performed in $21(87.5 \%)$ and $3(12.5 \%)$ patients, respectively. Additionally, rectal resection with lateral lymph node dissection was performed in 8 patients. The median operating time was 520 (range, 230769) $\mathrm{min}$, and the median blood loss was 50 (range, 5-500) g. The cohort included 9 (37.5\%), 7 (29.2\%),

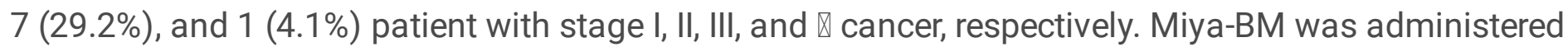
orally in $8(33 \%)$ patients. AL and ileus occurred in $4(16.7 \%)$ and $1(4.1 \%)$ patient, respectively, whereas $12(50 \%)$ patients suffered intestinal fluid loss due to high-output ileostomy. The median number of postoperative days in hospital was 14 (range, 10-52) days.

The study patients were categorized to the high-output $(n=12)$ and the low-output $(n=12)$ groups. There were no significant differences in sex, age, body mass index, preoperative therapy, surgical procedure, operation time, blood loss, duration of antibiotic use, Miya-BM use, and postoperative complications such as $\mathrm{AL}$ and postoperative ileus between the two groups (Table 2).

The comparison of the a-diversity between the high-output and low-output groups using several indices, including the observed species, PD whole index, and Chao1 index, revealed that there were no statistically significant differences in the a-diversity between the two groups (Fig. 1A). The overall community structure of the gut microbiota of both groups was evaluated using $\beta$-diversity indices calculated by the weighted UniFrac distance (Fig. 1B-1C). The patients in the high-output group exhibited the highest UniFrac distances by Bonferroni test for multiple comparisons, indicating that the microbiota composition of the high-output group was significantly different than that of the low-output group.

The differences in the gut microbial structure were taxonomically evaluated at the phylum level (Fig. 2A). The major bacteria were Bacteroidetes $(14.8 \%$ and $0.5 \%$ in high-output and low-output groups, 
respectively; $p=0.01)$, Firmicutes $(64.9 \%$ and $70.3 \%$ in high-output and low-output groups, respectively; $p$ $=0.63$ ), Proteobacteria ( $15.5 \%$ and $25.9 \%$ in high-output and low-output groups, respectively; $p=0.95$ ), Fusobacterium ( $2.5 \%$ and $0.1 \%$ in high-output and low-output groups, respectively; $p=0.73$ ), and Actinobacteria (2.0\% and $1.8 \%$ in high-output and low-output groups, respectively; $p=0.68)$.

The taxonomic changes in the microbial communities in the high-output group of patients were evaluated at the species level as well. As shown in Fig. 2B, the percentage of $C$. butyricum was higher $(p=0.01)$ and the percentage of Parabacteroides gordonii was lower $(p=0.07)$ in the low-output group. As $C$. butyricum is the main component of Miya-BM, secondary analysis was performed by categorizing patients not treated with Miya-BM into those with high output $(n=9)$ and those with low output $(n=7)$. There were no significant between-group differences in sex, age, body mass index, preoperative therapy, surgical procedure, operation time, blood loss, duration of antibiotic use, disease stage, and postoperative complications such as AL and postoperative ileus (Table 3 ). The comparison of the $\alpha$ - and $\beta$-diversity using the indices indicated above revealed that there were no statistically significant differences between the high-output and low-output patients with ileostomy who were not treated with Miya-BM (Fig. 3A-C).

In the subset of patients not treated with Mya-BM, the differences in the gut microbial structure between the high-output and the low-output groups were taxonomically evaluated at the phylum level (Fig. 4A). The major bacteria were Bacteroidetes $(16.8 \%$ and $0.1 \%$ in high-output and low-output groups, respectively; $p=0.03)$, Firmicutes $(66 \%$ and $61.9 \%$ in high-output and low-output groups, respectively; $p=$ 0.63 ), Proteobacteria ( $14.3 \%$ and $32.9 \%$ in high-output and low-output groups, respectively; $p=0.95$ ), and Actinobacteria ( $2.4 \%$ and $2.6 \%$ in high-output and low-output groups, respectively; $p=0.87$ ). Furthermore, as shown in Fig. $4 \mathrm{~B}$, there were no significant differences in the taxonomic changes of the microbial communities between the high-output and low-output patients not treated with Miya-BM. Although not statistically significant, the percentage of Corynebacterium durum was lower in the low-output group than in the high-output group among the patients not treated with Miya-BM $(p=0.09)$.

Overall, these data indicate that Miya-BM might be one of the reasons underlying the control of highoutput diarrhea and that dysbiosis of the gut microbiota might be associated with the intestinal fluid loss due to high-output ileostomy.

\section{Discussion And Conclusion}

The current study represents the first assessment of the bacterial composition in patients with highoutput stomas using next-generation sequencing. Excessive output from the stoma and related electrolyte abnormalities are precursors of dehydration and renal dysfunction. ${ }^{17}$ In clinical practice, testing for $C$. difficile is important in detecting the underlying cause of high output due to the increasing number of diarrhea cases associated with $C$. difficile during hospitalization, which affects 3-10 patients per 1000 hospitalizations. ${ }^{18}$ Generally, long-term antibiotic use has been widely documented to disturb the gut microbiota and to associate with $C$. difficile infection. ${ }^{18}$ However, the current study did not show a correlation between high-output stomas and CDI. 
Previous studies have shown that relative abundance of microbes is a function of location along the cephalocaudal axis of the distal gut. Bacteroides and Firmicutes were isolated from the terminal ileum. ${ }^{19,20}$ In the current study, we found decreased $\beta$-diversity in the low-output patients and that the percentage of Bacteroides was very low in the low-output group even though Bacteroides are the major intestinal commensal bacteria. However, given that cefmetazole administered in all patients was not effective against some Bacteroides species, these findings suggest the emergence of a pathological flora in the low-output group. Bacteroides have a stronger ability to induce the production of immunoglobulin A from the lymphocytes in Peyer's patches, providing protection against inflammation. ${ }^{21}$ Low-output stomas may be pathogenic due to the loss of Bacteroides.

Studies previously have demonstrated that Miya-BM is effective in the prevention and reduction of antibiotic-associated diarrhea. ${ }^{22}$ Miya-BM has also been used in antibiotic-associated diarrhea during Helicobacter pylori eradication therapy. ${ }^{23}$ There has been an increase in the studies demonstrating an interaction between the intestinal microbiota and a wide variety of conditions including obesity, cardiometabolic diseases, inflammatory bowel disease (IBD), CDI, irritable bowel syndrome, insulin resistance, multiple sclerosis, and idiopathic thrombocytopenic purpura. Fecal microbiota transplantation appears to be a safe and promising treatment for IBD and recurrent CDI. Well- designed randomized controlled trials are needed to establish the efficacy of fecal microbiota transplantation for other diseases as well. Future studies should analyze the composition of the small intestinal and fecal microbiota before and after fecal microbiota transplantation. ${ }^{24}$ The current study results suggest that probiotics might be a candidate approach that might be utilized to control issues associated with high-output stomas following ileostomy. Whether the flora was pathological could not be determined in either group. There was a loss of Bacteroides in the terminal ileum of the low-output patients, whereas $C$. butyricum was absent in the intestinal fluid of high-output patients. Furthermore, the analysis of the cohort after the exclusion of patients treated with Miya-BM indicated additional important differences associated with the output. This result is in line with previous studies that have reported that the differences in the composition and diversity of the gut microbiota are related to the specific food products, dietary habits, geographical origin, and antibiotics. ${ }^{25,26}$

The current study has several limitations, including the small number of patients and the limited investigation of the influence of diet as well as the impact of potential biases including the uneven distribution of sex, previous antibiotic use, dietary habits, and geographical origin between the groups. The findings of the current study merit further investigation in well-designed, large, confirmatory studies.

In conclusion, this pilot study provides the first evidence of a correlation between the gut microbiota and the pathogenesis of high-output stoma compared with that of low-output stoma.

\section{Abbreviations}


$\mathrm{AL}$, anastomotic leakage; CDI, Clostridium difficile infection; IBD, inflammatory bowel disease; ISR, intersphincteric resection; LAR, low anterior resection;

\section{Declarations}

\section{Ethics approval and consent to participate}

This study was performed in accordance with the ethical standards of the Committee on Human Experimentation of the study institution (Institutional Review Board No.16-124)

\section{Consent for publication}

Not applicable.

\section{Availability of data and materials}

The datasets used and analysed during the current study are available from the corresponding author on reasonable request.

\section{Competing interests}

The authors declare no conflict of interest.

\section{Funding}

This study was supported by Miyarisan Pharmaceutical Co., Ltd.Japan (to K.S.). The funders had no role in the study design, data collection and analysis, decision to publish, and preparation of the manuscript.

\section{Authors' contributions}

Study concept and design: HM, HK, and SM. Data acquisition: HM. Analysis and interpretation of data: $\mathrm{HM}, \mathrm{SM}, \mathrm{YK}, \mathrm{MK}, \mathrm{KS}$, and MT. Drafting of the manuscript: HM, SM. Critical revision of the manuscript: SM, KS.

\section{Acknowledgements}

The authors thank Yasutoshi Kuroki, Seiya Higashi, Oka Kentaro, and Motomichi Takahashi for kindly providing technical assistance \& statistical analysis.

\section{References}

1.

Shiomi $\mathrm{A}$, Ito $\mathrm{M}$, Maeda $\mathrm{K}$, et al. Effects of a diverting stoma on symptomatic anastomotic leakage after low anterior resection for rectal cancer: a propensity score matching analysis of 1,014 consecutive patients. J Am Coll Surg. 2015;220:186-94. 
Munakata S, Ito S, Sugimoto K, et al. Defunctioning loop ileostomy with restorative proctocolectomy for rectal cancer: Friend or foe? J Anus Rectum Colon. 2017;1:136-40.

3.

Shabbir J, Britton DC. Stoma complications: a literature overview. Colorectal Dis Off J Assoc Coloproctol Gr Br Irel. 2010;12:958-64.

4.

Beck-Kaltenbach N, Voigt K, Rumstadt B. Renal impairment caused by temporary loop ileostomy. Int J Colorectal Dis. 2011;26:623-6.

5 .

Nightingale J, Woodward JM, Small B. Nutrition Committee of the British Society of G. Guidelines for management of patients with a short bowel. Gut. 2006;55(Suppl 4):iv1-12.

6.

Lynch SV, Pedersen O. The Human Intestinal Microbiome in Health and Disease. N Engl J Med. 2016;375:2369-79.

7.

Sartor RB, Wu GD. Roles for Intestinal Bacteria, Viruses, and Fungi in Pathogenesis of Inflammatory Bowel Diseases and Therapeutic Approaches. Gastroenterology. 2017;152:327-39.e4.

8.

Hata $\mathrm{H}$, Yamaguchi T, Hasegawa S, et al. Oral and parenteral versus parenteral antibiotic prophylaxis in elective laparoscopic colorectal surgery (JMTO PREV 07 - 01): A phase 3, multicenter, open-label, randomized trial. Ann Surg. 2016;263:1085-91.

9.

Sadahiro S, Suzuki T, Tanaka A, et al. Comparison between oral antibiotics and probiotics as bowel preparation for elective colon cancer surgery to prevent infection: prospective randomized trial. Surgery. 2014;155:493-503.

10.

Law WL, Choi HK, Lee YM, Ho JW, Seto CL. Anastomotic leakage is associated with poor long-term outcome in patients after curative colorectal resection for malignancy. J Gastrointest Surg. 2007;11:815 .

11.

Rahbari NN, Weitz J, Hohenberger W, Heald RJ, Moran B, Ulrich A, et al. Definition and grading of anastomotic leakage following anterior resection of the rectum: a proposal by the International Study Group of Rectal Cancer. Surgery. 2010;147:339-51.

12.

Horan TC, Gaynes RP, Martone WJ, Jarvis WR, Emori TG. CDC definitions of nosocomial surgical site infections, 1992: a modification of CDC definitions of surgical wound infections. Infect Control Hosp Epidemiol. 1992;13:606-8.

13. 
Law WL, Chu KW, Tung PH. Laparoscopic colorectal resection: a safe option for elderly patients. J Am Coll Surg. 2002;195:768-73.

14.

Trotti A, Colevas AD, Setser A, et al. CTCAE v3.0: development of a comprehensive grading system for the adverse effects of cancer treatment. Semin Radiat Oncol. 2003;13:176-81.

15.

Hayden DM, Pinzon MC, Francescatti AB, et al. Hospital readmission for fluid and electrolyte abnormalities following ileostomy construction: preventable or unpredictable? J Gastrointest Surg. 2013;17:298-303.

16.

Arenas Villafranca JJ, Lopez-Rodriguez C, Abiles J, Rivera R, Gandara Adan N, Utrilla Navarro P. Protocol for the detection and nutritional management of high-output stomas. Nutr J. 2015;14:45.

17.

Caricato M, Ausania F, Ripetti V, Bartolozzi F, Campoli G, Coppola R. Retrospective analysis of long-term defunctioning stoma complications after colorectal surgery. Colorectal Dis. 2007;9:559-61.

18.

Johal SS, Hammond J, Solomon K, James PD, Mahida YR. Clostridium difficile associated diarrhoea in hospitalised patients: onset in the community and hospital and role of flexible sigmoidoscopy. Gut. 2004;53:673-7.

19.

Peterson DA, Frank DN, Pace NR, Gordon JI. Metagenomic approaches for defining the pathogenesis of inflammatory bowel diseases. Cell Host Microbe. 2008;3:417-27.

20.

Hattori M, Taylor TD. The human intestinal microbiome: a new frontier of human biology. DNA Res. 2009;16:1-12.

21.

Yanagibashi T, Hosono A, Oyama A, et al. Bacteroides induce higher IgA production than Lactobacillus by increasing activation-induced cytidine deaminase expression in B cells in murine Peyer's patches. Biosci Biotechnol Biochem. 2009;73:372-7.

22.

Seki H, Shiohara M, Matsumura T, et al. Prevention of antibiotic-associated diarrhea in children by Clostridium butyricum MIYAIRI. Pediatr Int. 2003;45(1):86-90.

23.

Imase K, Takahashi M, Tanaka A, Tokunaga K, Sugano H, Tanaka M, et al. Efficacy of Clostridium butyricum preparation concomitantly with Helicobacter pylori eradication therapy in relation to changes in the intestinal microbiota. Microbiol Immunol. 2008;52:156-61.

24.

Smits LP, Bouter KE, de Vos WM, Borody TJ, Nieuwdorp M. Therapeutic potential of fecal microbiota transplantation. Gastroenterology. 2013;145:946-53.

25 .

Page 10/16 
Senghor B, Sokhna C, Ruimy R, Lagier J-C. Gut microbiota diversity according to dietary habits and geographical provenance. Hum Microbiome J. 2018;7-8:1-9.

26.

lizumi T, Battaglia T, Ruiz V, Perez Perez GI. Gut microbiome and antibiotics. Arch Med Res. 2017;48:72734.

\section{Tables}

Due to technical limitations, tables are only available as a download in the supplemental files section.

\section{Figures}


A
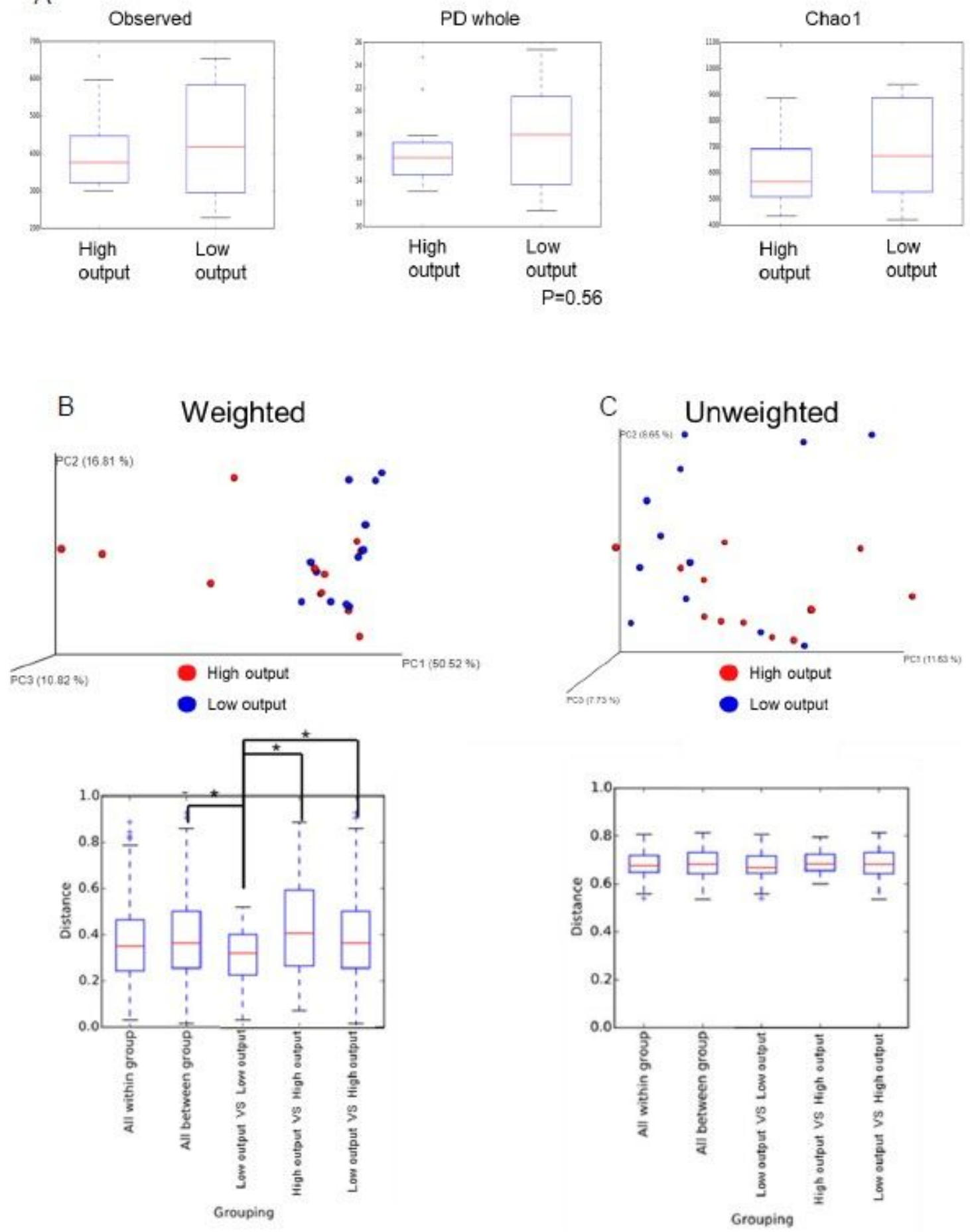

\section{Figure 1}

The microbial communities of the high-output and low-output groups in patients with ileostomy. (A) adiversity indices and (B) weighted and (C) unweighted principal coordinate analysis ( $\mathrm{pCoA}$ ) of $\beta$-diversity measures for all samples. Values represent means \pm standard error of the mean (SEM). ${ }^{*} p<0.05,{ }^{*} p<$ $0.01, \star \star \star x p<0.001$ 
A
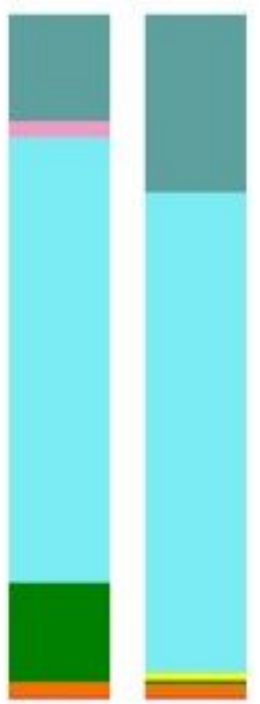

High output

$\mathrm{n}=12$

Proteobacteria

Fusobacteria

Firmicutes

Bacteroidetes

Actinobacteria
Proteobacteria
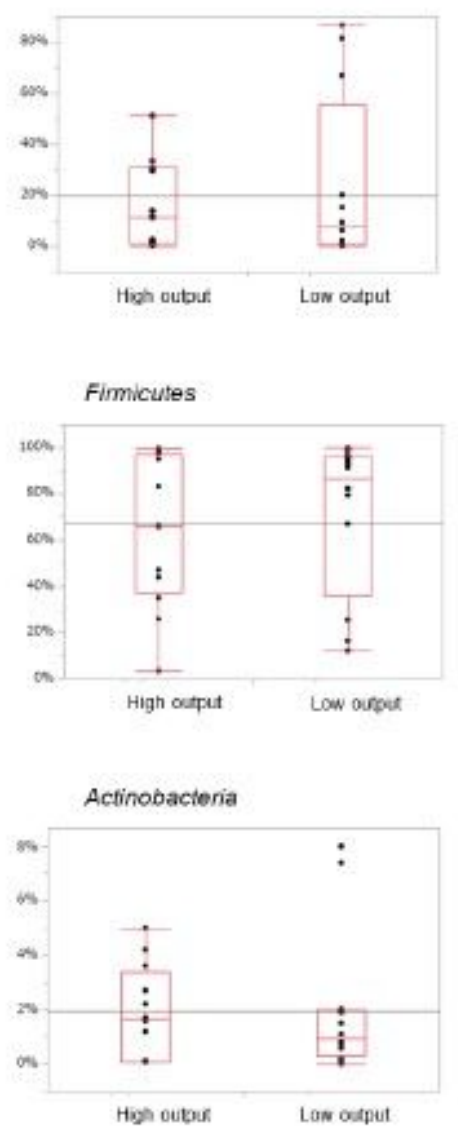

Fusobacteria
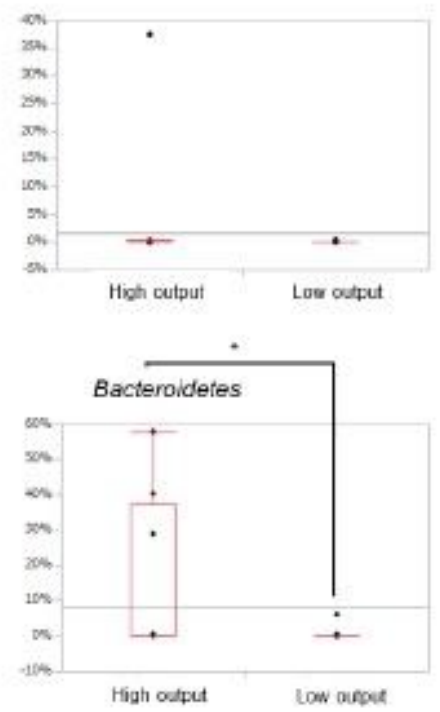

B
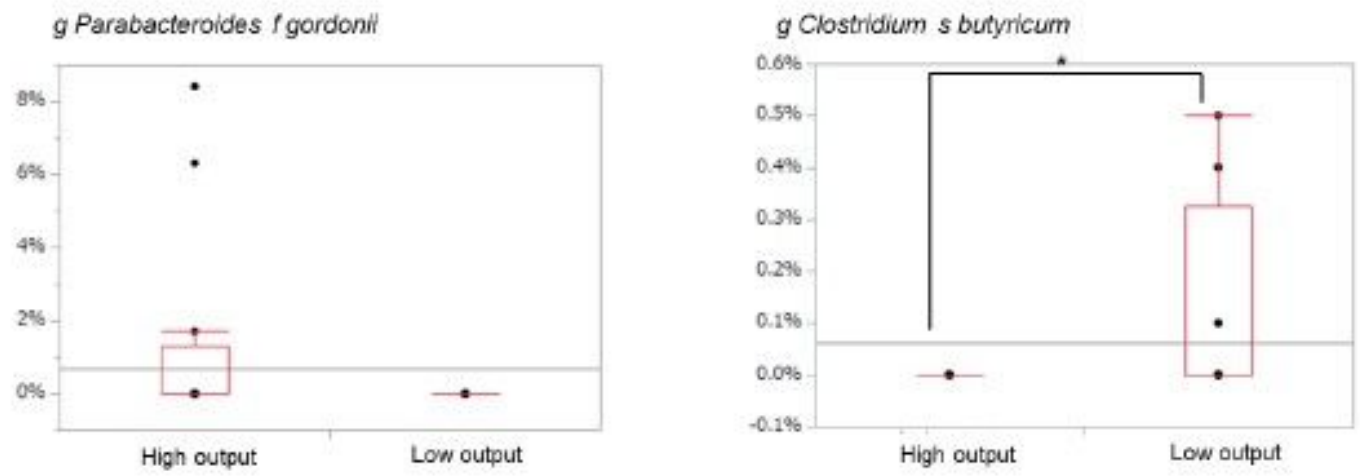

\section{Figure 2}

Comparative analyses of the taxonomic composition of the microbial community at the phylum $(A)$ and species (B) levels. Representative bar charts with significant differences between the groups are presented. Values represent means \pm SEM. ${ }^{\star} p<0.05,{ }^{\star \star} p<0.01,{ }^{\star \star *} p<0.001$ 
A
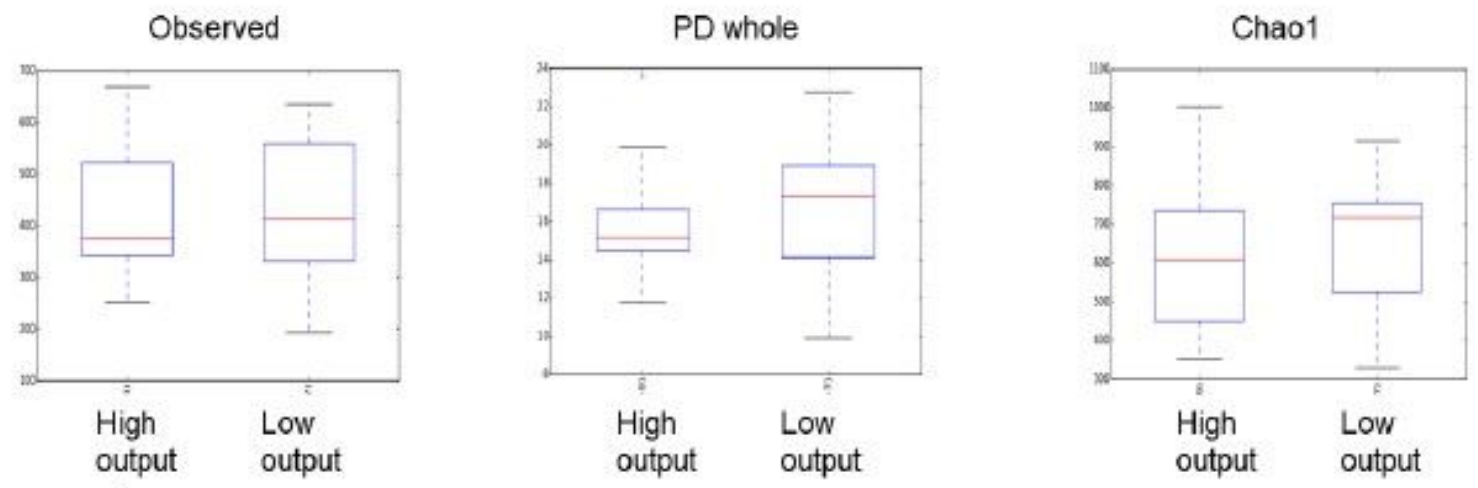

B Weighted
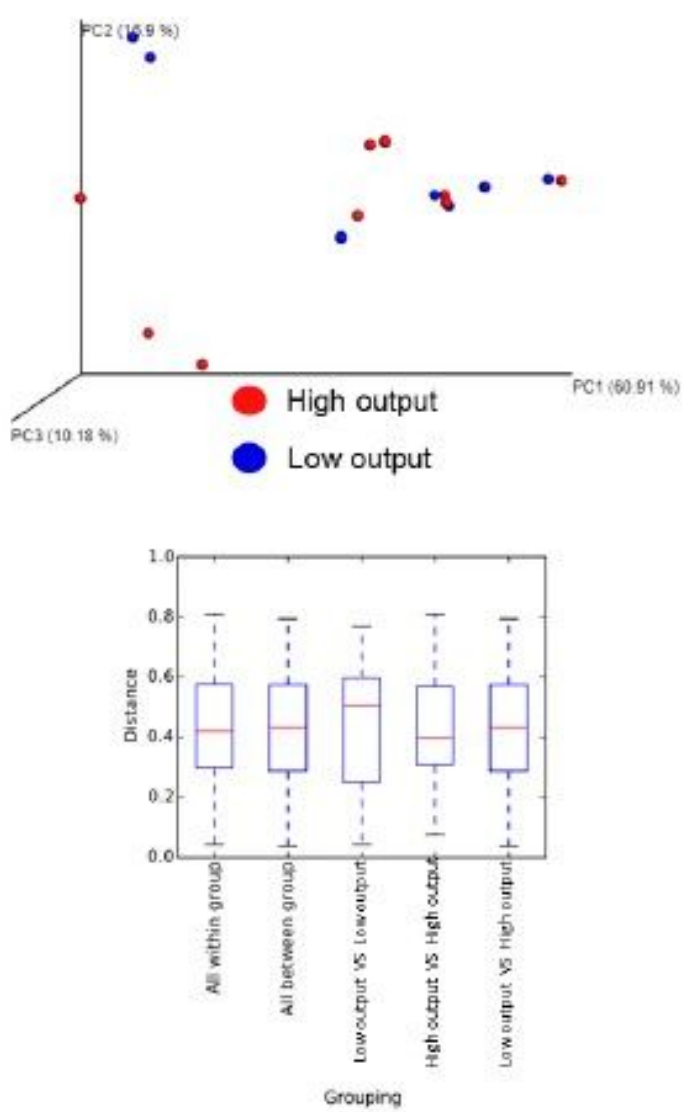

c Unweighted
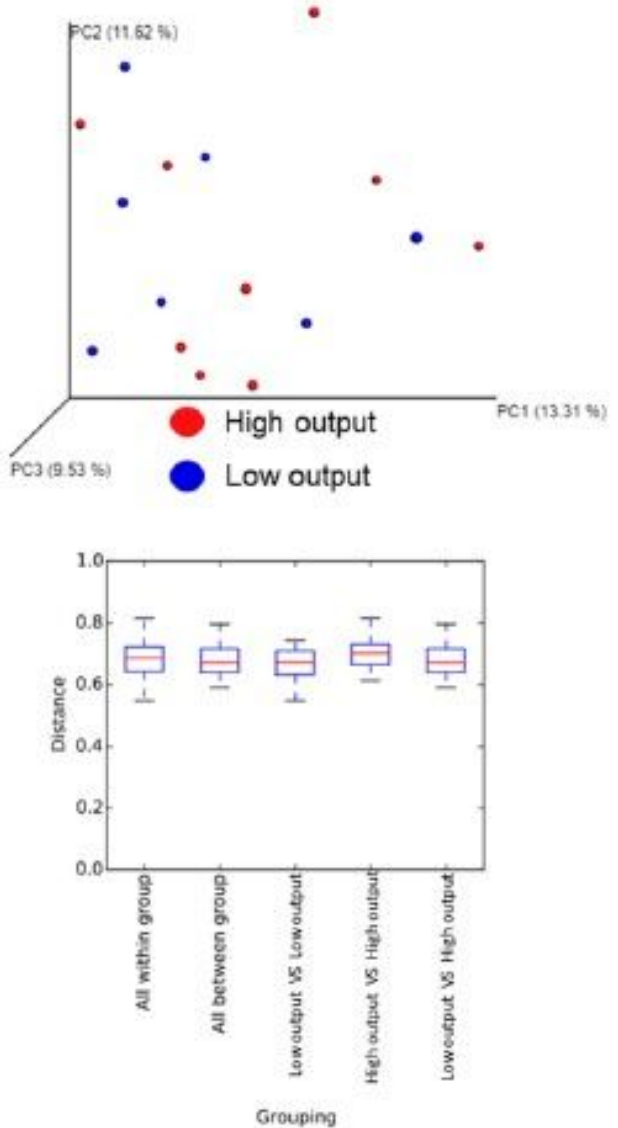

\section{Figure 3}

The microbial communities of the high-output and low-output groups in patients with ileostomy not treated with Miya-BM. (A) a-diversity indices and (B) weighted and (C) unweighted pCoA of $\beta$-diversity measures for all samples. Values represent means \pm SEM. ${ }^{\star} p<0.05,{ }^{\star \star} p<0.01,{ }^{\star \star \star} p<0.001$ 
A
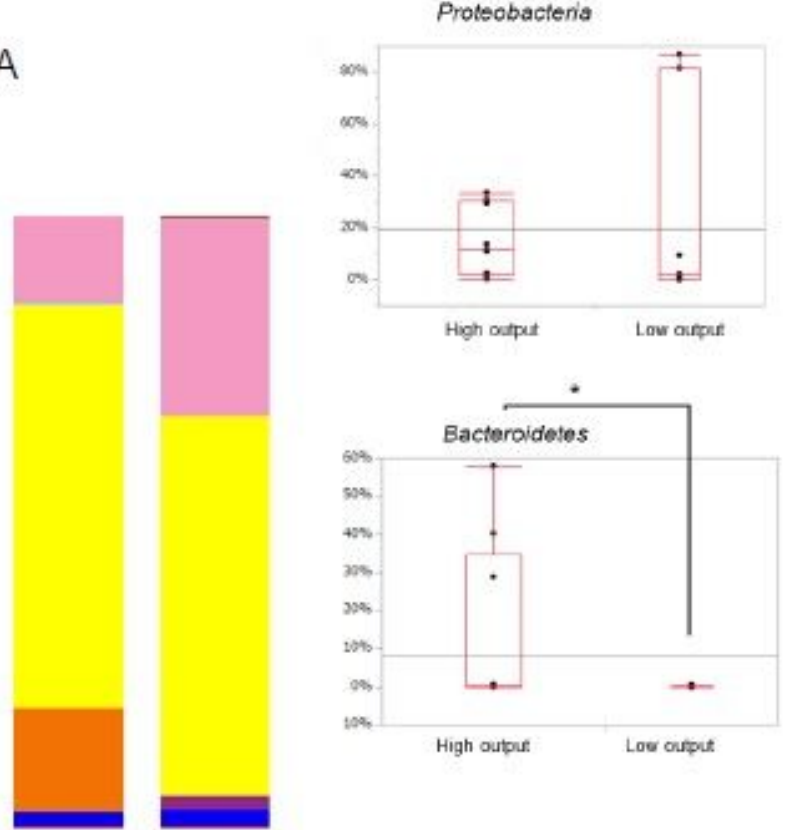

High

output

$n=9$

Proteobacteria

Firmicutes

Bacteroidetes

- Actinobacteria

B

$g$ Corynebacteriaceae s durum

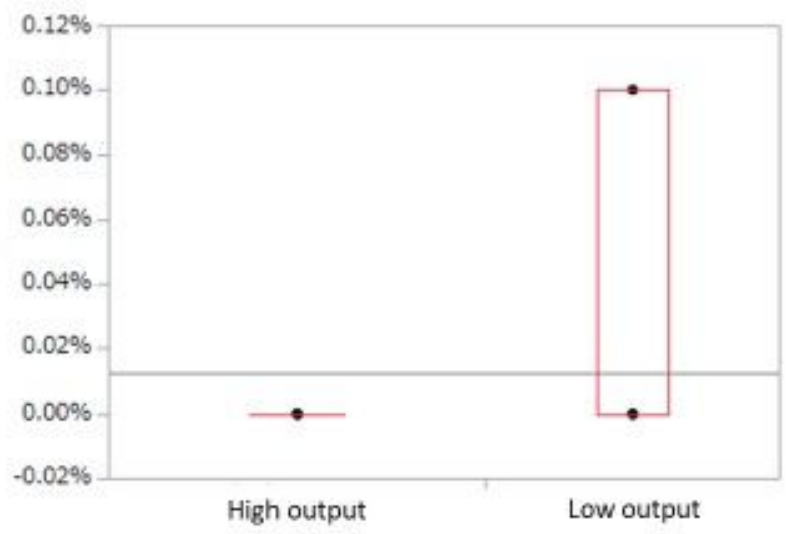

Low output

$n=7$

ia
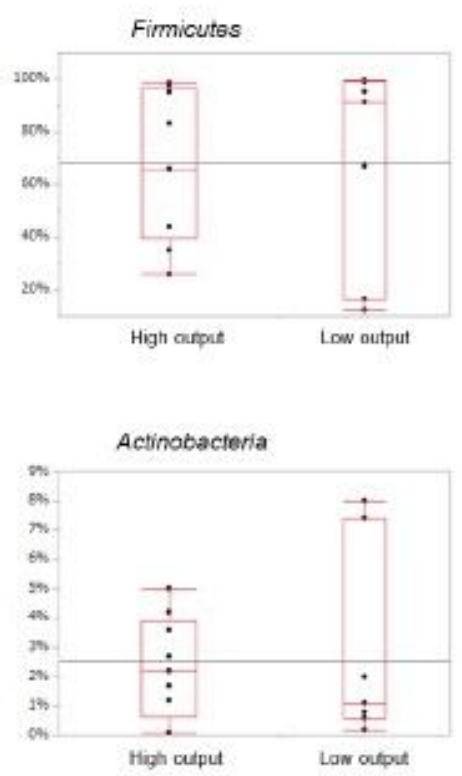


\section{Supplementary Files}

This is a list of supplementary files associated with this preprint. Click to download.

- Table1.pptx

- Table3.pptx

- Table2.pptx 